\title{
Fermented Soy Beverage Q-CAN Plus Consumption Improves Serum Cholesterol and Cytokines
}

\author{
Suyavaran Arumugam, ${ }^{1}$ Evangelos Dioletis, ${ }^{1}$ Ricardo Paiva, ${ }^{1}$ Maxine R. Fields, ${ }^{2}$ \\ Theresa R. Weiss, ${ }^{2}$ Eric R. Secor, ${ }^{3}$ and Ather Ali ${ }^{2}$ \\ ${ }^{1}$ Internal Medicine (Digestive Diseases); ${ }^{2}$ Department of Pediatrics (General Pediatrics); Yale University School of Medicine, \\ New Haven, Connecticut, USA. \\ ${ }^{3}$ Hartford Hospital and University of Connecticut, Hartford, Connecticut, USA.
}

\begin{abstract}
Soy-based beverages are well recognized for their rich nutritional contents and positive health benefits. However, there is little information regarding the composition of various commercially available soy-based beverages and uncertainty among patients regarding the utility of fermented soy products. Current study evaluates the health benefits of QCAN $^{\circledR}$ Plus — an easily available fermented soy drink. This study was performed in lean $(n=10)$ and obese $(n=10)$ subjects. The subjects were observed during pre-soy (weeks $-2,-1$, and 0 ), on-soy (weeks 1, 2, 3, and 4), and post-soy (weeks 6, 8, 10, and 12) periods. The serum samples during these visits were subjected to lipid profile analysis and multiplex assay for cytokines. The results revealed that total cholesterol and low-density lipoprotein (LDL) cholesterol levels were significantly reduced in both lean and obese individuals during on-soy $(P \leq .05)$. Furthermore, cytokines such as platelet-derived growth factor (PDGF) AA and AB/BB were significantly lowered on-soy compared with pre-soy $(P \leq .05)$ in lean subjects and PDGF AA, IL-1RA, and GMCSF were significantly reduced on-soy $(P \leq .05)$ in obese subjects. In addition, a qualitative and quantitative analysis of the Q-CAN Plus by a third-party laboratory confirmed its chemical and microbial safety. Our preliminary study on Q-CAN Plus ensures its safety for consumption and highlights its hypolipidemic and suppressive effect on certain cytokines. These observations and relevant studies in future might guide clinicians in future to consider Q-CAN Plus as a therapeutic nutritional supplement.
\end{abstract}

\section{KEYWORDS: • cholesterol $\bullet$ cytokines $\bullet$ fermented soy drink $\bullet$ hyperlipidemia $\bullet$ isoflavones $\bullet$ obesity}

\section{INTRODUCTION}

$\mathbf{S}^{\mathrm{s}}$ OYBEAN IS AN INTEGRAL PART of most southeast Asian cuisines since ancient times and has recently been adopted by the western world for its nutritional significance. Several active components have been identified and characterized in soy. ${ }^{1,2}$ Natural isoflavones in soy are conjugated to sugars, thereby restricting their dietary absorption. Fermentation of soy increases aglycone form of isoflavones thus increasing their bioavailability. ${ }^{3}$ Fermentation of soy has also been demonstrated to improve its vitamin and mineral contents, which further improves the nutritional value, ${ }^{4,5}$ and is thought to contribute to the cholesterol lowering, antidiabetic and cardioprotective effects of soy-based food products. ${ }^{6-8}$ However, it has been difficult to translate these experimental results into practical dietary advice because there is a paucity of reliable data on the composition of soy

Manuscript received 23 May 2019. Revision accepted 30 August 2019.

Address correspondence to: Suyavaran Arumugam, PhD, Internal Medicine (Digestive Diseases), Yale University School of Medicine, New Haven, CT 06520, USA, E-mail: arumugam.suyavaran@yale.edu products in studies and patient confusion regarding selection of fermented soy products. Therefore, our goal was to test both the quality of widely available fermented soy product and its ability to reduce serum cholesterol and antiinflammatory biomarkers. With a growing prevalence of obesity, we aimed to evaluate whether these were applicable to lean and obese individuals. ${ }^{9}$

\section{METHODS}

We performed a prospective study of lean (mean BMI $\left.21.9 \mathrm{~kg} / \mathrm{m}^{2}\right)(n=10)$ and obese (mean BMI $\left.31.9 \mathrm{~kg} / \mathrm{m}^{2}\right)(n=$ 10) individuals without a known diagnosis of hyperlipidemia (Yale institutional review board HIC\# 1507016139; Clinical Trials.gov Identifier: NCT02656056) (Table 1). The distinctive smell and taste of Q-CAN ${ }^{\circledR}$ fermented soy drink made it impractical to have a placebo. The intervention consisted of twice daily consumption of $237 \mathrm{~mL}$ of QCAN for 4 weeks. Weeks $-2,-1$, and 0 were pre-soy, weeks $1,2,3$, and 4 were on-soy, and weeks $6,8,10$, and 12 were post-soy. Two hundred and thirty-seven milliliter of fermented soy (Q-CAN) was dispensed at week 0 and was consumed twice daily for 4 weeks. Blood was collected 
Table 1. Demographic Characteristics of Subjects Included in the Evaluation of Fermented Soy Beverage Q-CAN Plus for Its Nutraceutical Benefits

\begin{tabular}{lcc}
\hline Demographic characteristics & Lean $(\mathrm{n}=10)$ & Obese $(\mathrm{n}=10)$ \\
\hline Gender $(n)$ & 7 & 3 \\
$\quad$ Female & 3 & 7 \\
$\quad$ Male & $32.2 \pm 12.23$ & $44.5 \pm 13.75$ \\
Age (mean, SD) & $136.25 \pm 22.42$ & $221.90 \pm 29.13$ \\
Weight (mean, SD) & $21.85 \pm 2.01$ & $33.90 \pm 1.75$ \\
BMI (mean, SD) & & \\
Race (n) & 8 & 5 \\
White & 1 & 2 \\
Black & 1 & 3 \\
Unknown & & 7 \\
Ethnicity ( $n$ ) & 6 & 2 \\
$\quad$ Non-Hispanic & 4 & 1 \\
Hispanic or Latino & 0 & Depression (1) \\
Unknown & & Type 2 DM (1) \\
Comorbidities/diagnoses & & Hypertension (2) \\
& & \\
& &
\end{tabular}

DM, diabetes mellitus; SD, standard deviation.

during weeks $-2,0,2,4,6,8,10$, and 12 . Serum from blood samples were subjected to quantification of lipid parameters, including total cholesterol, low-density lipoprotein (LDL) cholesterol, and high-density lipoprotein (HDL) cholesterol, at a clinical chemistry laboratory. Cytokine levels were assayed using Human Magnetic Luminex ${ }^{\circledR}$ - Human XL cytokine assay (R\&D Systems, MN) and the multiplex analysis was performed at CytoPlex core facility, division of reproductive sciences, Yale School of Medicine, Yale University. The kit analyzes a list of 45 analytes, of which only CCL-2, CCL-4, CCL-5, CCL-11, CCL-20, FLT-3 ligand, CXCL-2, CXCL-3, CXCL-10, platelet-derived growth factor (PDGF) AB/BB, AA, IL-1RA, IL-10, PDL1, VEGF, GMCSF, and TRAIL were of detectable levels and were expressed as $\mathrm{pg} / \mathrm{mL}$ of serum. The data were statistically analyzed by one-way ANOVA with multiple comparisons (significance of $P \leq .05$ ) for the three therapeutic phases of pre-soy, on-soy, and post-soy. A combined analysis of obese and lean groups was performed followed by separate analysis. In addition to the clinical study, Q-CAN product was also analyzed by a third-party laboratory for the presence of heavy metals (arsenic, cadmium, lead, and mercury), residual solvents and microbes to ensure its safety for consumption.

\section{RESULTS AND DISCUSSION}

The combined analysis of lean and obese subjects showed that HDL cholesterol (pre $=45.2632$; on $=40.1053$ ), total cholesterol (pre $=161.421$; on $=146.789)$, and LDL cholesterol $($ pre $=95.8947$; on $=82.4211)$ were significantly decreased $(P<.05)$ (Table 2). Further separate analysis of lean and obese subjects was performed. Both lean and obese subjects had a significant decrease in total cholesterol levels
Table 2. Data (Mean) from Lean and Obese Groups Were Combined And Analyzed by One-Way ANOVA With Multiple Comparisons

\begin{tabular}{lccc}
\hline Parameter & Pretreatment & During & $\mathrm{P}$ \\
\hline Lipid profile & & & \\
Triglycerides & 70 & 90.364 & .0677 \\
HDL & 45.2632 & 40.1053 & $.0107^{*}$ \\
Total cholesterol & 161.421 & 146.789 & $.0352^{*}$ \\
LDL & 95.8947 & 82.4211 & $.0308^{*}$ \\
Cytokines & & & \\
PDGF AB/BB & 520.654 & 361.559 & .0626 \\
PDGF AA & 1380.97 & 957.841 & .0703 \\
IL-TRA & 760.26 & 295.764 & .1976 \\
VEGF & 81.247 & 75.142 & .2295 \\
CCL20 & 14.1938 & 17.3209 & .8488 \\
CCL4 & 99.4747 & 92.707 & .892 \\
\hline *P<.05. & \multicolumn{4}{l}{} \\
HDL, high-density lipoprotein; LDL, low-density lipoprotein; & PDGF, \\
platelet-derived growth factor. &
\end{tabular}

on-soy of as compared with pre-soy (mean lean, pre $=167$ $\mathrm{mg} / \mathrm{dL}$, on $=151.5, P=.0343$; obese, pre $=155.2$, on $=141.6$, $P=.0256$ ) and LDL cholesterol (mean lean pre $=102.7$, on $=80.3, P=.0181$, mean obese, pre $=96.1$, on $=82.4$, $P=.0412$ ) (Fig. 1). Post-soy total cholesterol and LDL cholesterol values trended toward baseline. No significant changes were observed in HDL and triglyceride. We also compared the percentage change in lipid parameters using pre-soy as a reference. Q-CAN Plus consumption in lean subjects resulted in a 7\% decrease in LDL, and 9\% reduction in total cholesterol levels, whereas in obese subjects resulted in a $16 \%$ reduction in LDL, and a $7 \%$ reduction in total cholesterol levels $(P<.05)$. The percentage change post-soy was not significant.

In lean subjects on-soy PDGF $\mathrm{AA}$ and $\mathrm{AB} / \mathrm{BB}$ was significantly lower as compared with pretreatment, and this was sustained post-soy (PDGF AA mean, $\mathrm{pre}=1355 \mathrm{pg} / \mathrm{mL}$, on $=913 \mathrm{pg} / \mathrm{mL}$ and post $=746, P<.05 ; \quad$ PDGF $\mathrm{AB} / \mathrm{BB}$ mean, pre $=611$, on $=403$ and post $=313 \mathrm{pg} / \mathrm{mL}, P<.05$ ) (Fig. 2). In obese only PDGF AA levels showed significant reduction on-soy and returned to baseline post-soy (PDGF AA mean pre $=922 \mathrm{pg} / \mathrm{mL}$ and on $=694 \mathrm{pg} / \mathrm{mL}$; PDGF $\mathrm{AB} / \mathrm{BB}$ mean in obese pretreatment $=267 \mathrm{pg} / \mathrm{mL}$ ) (Fig. 3). In obese subjects, the levels of IL-1RA and GMCSF were also significantly lowered on-soy as compared with their respective pre-soy phase levels (IL-1RA mean, pre $=474$ $\mathrm{pg} / \mathrm{mL}$ and on $=353 \mathrm{pg} / \mathrm{mL}, P<.05$; GMCSF mean, pre $=$ $14 \mathrm{pg} / \mathrm{mL}$ and on $=10 \mathrm{pg} / \mathrm{mL}, P<.05)$. Post-soy IL-1RA and GMCSF levels in obese subjects trended back to baseline. In addition, independent analysis from a third-party laboratory revealed that Q-CAN contained nutrients as stated on the label and was free of aflatoxins, microbial contaminants, solvents, and pesticides.

Our preliminary study reports that Q-CAN Plus, a widely available fermented soy drink, had hypolipidemic effect in obese and lean individuals, specifically on total and LDL cholesterol. Similarly, earlier meta-analysis by Taku et al., which involved 11 randomized control trials of soy intake in 

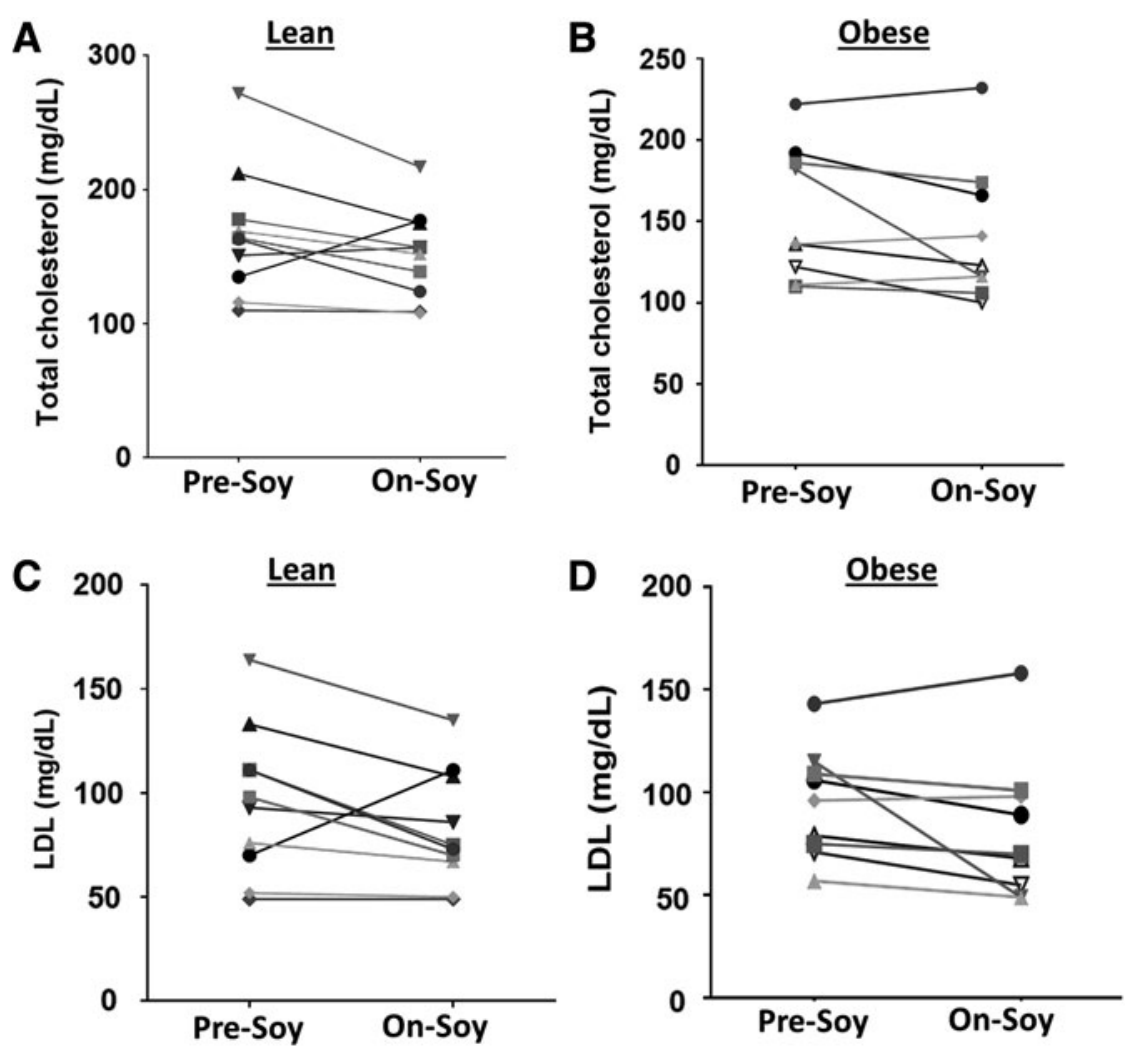

FIG. 1. Pre- and on-soy total $(\mathbf{A}, \mathbf{B})$ and LDL cholesterol $(\mathbf{C}, \mathbf{D})$ in lean and obese subjects. There was a significant $(P<.05)$ decrease in total and LDL cholesterol in lean and obese subjects when consuming fermented soy. LDL, lowdensity lipoprotein.

humans, had reported that soy isoflavones significantly reduced total cholesterol and LDL cholesterol with no changes in HDL cholesterol or triacyglycerol. ${ }^{10} \mathrm{We}$ observed that the levels of PDGF-AA were significantly lowered in Q-CAN Plus treated obese subjects. A study by Abderrahmani et al. showed that PDGF AA levels are increased in nonalcoholic fatty liver disease (NAFLD) and can contribute to insulin resistance-induced type-2 diabetes in NAFLD patients. ${ }^{11}$
Hence, Q-CAN Plus could be considered for dietary management in obese patients to prevent type- 2 diabetes under NAFLD conditions. The distinctive smell and taste of QCAN did not allow for the use of a control, and for this reason the three different phases of pre-, on-, and postQCAN were analyzed. The data of a reduction in serum cholesterol are consistent with earlier findings of a reduction in serum cholesterol with experimental soy products,

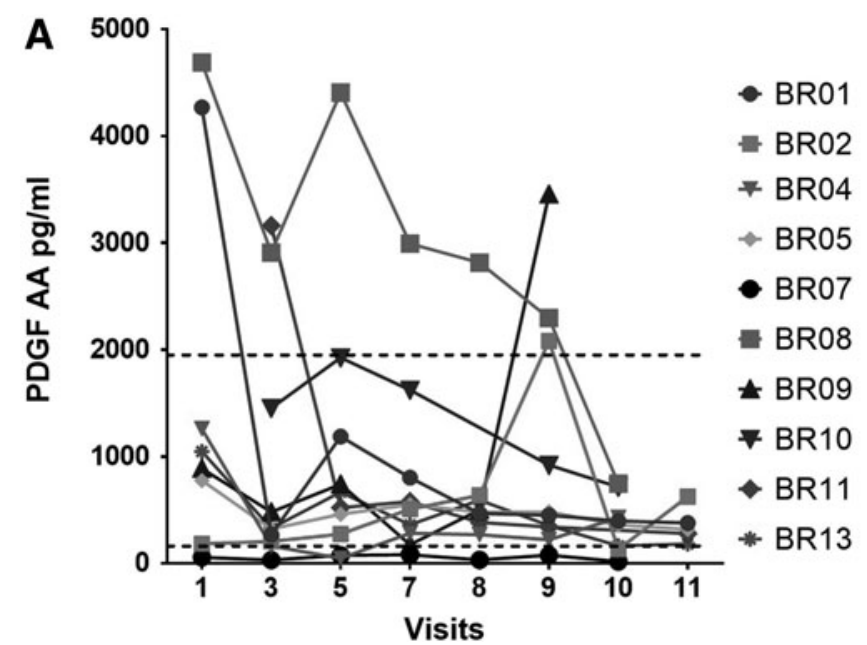

B

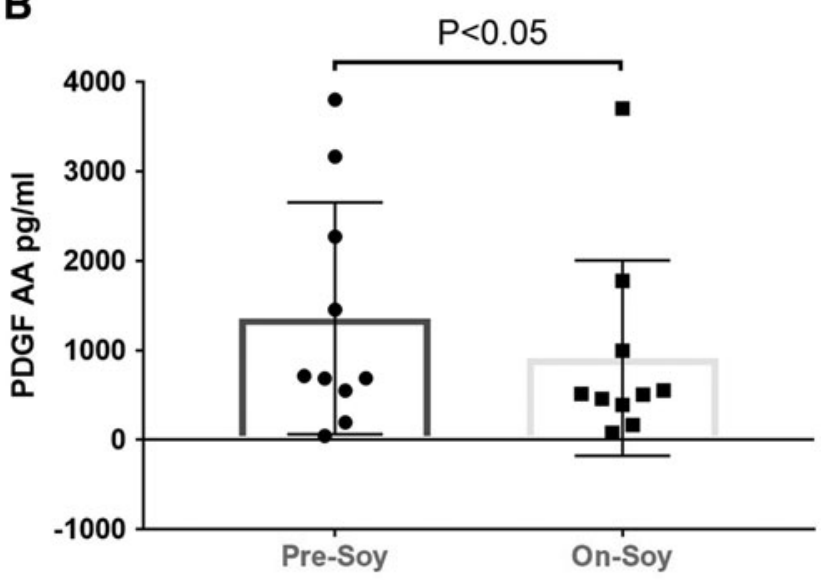

FIG. 2. (A) Plot graphs showing serum PDGF AA levels in lean subjects, pretreatment, and during treatment of Q-CAN Plus. (B) Histograms depicting mean \pm SD values of serum PDGF AA levels in lean subjects, pretreatment, and during treatment of Q-CAN Plus. PDGF, plateletderived growth factor; $\mathrm{SD}$, standard deviation. 

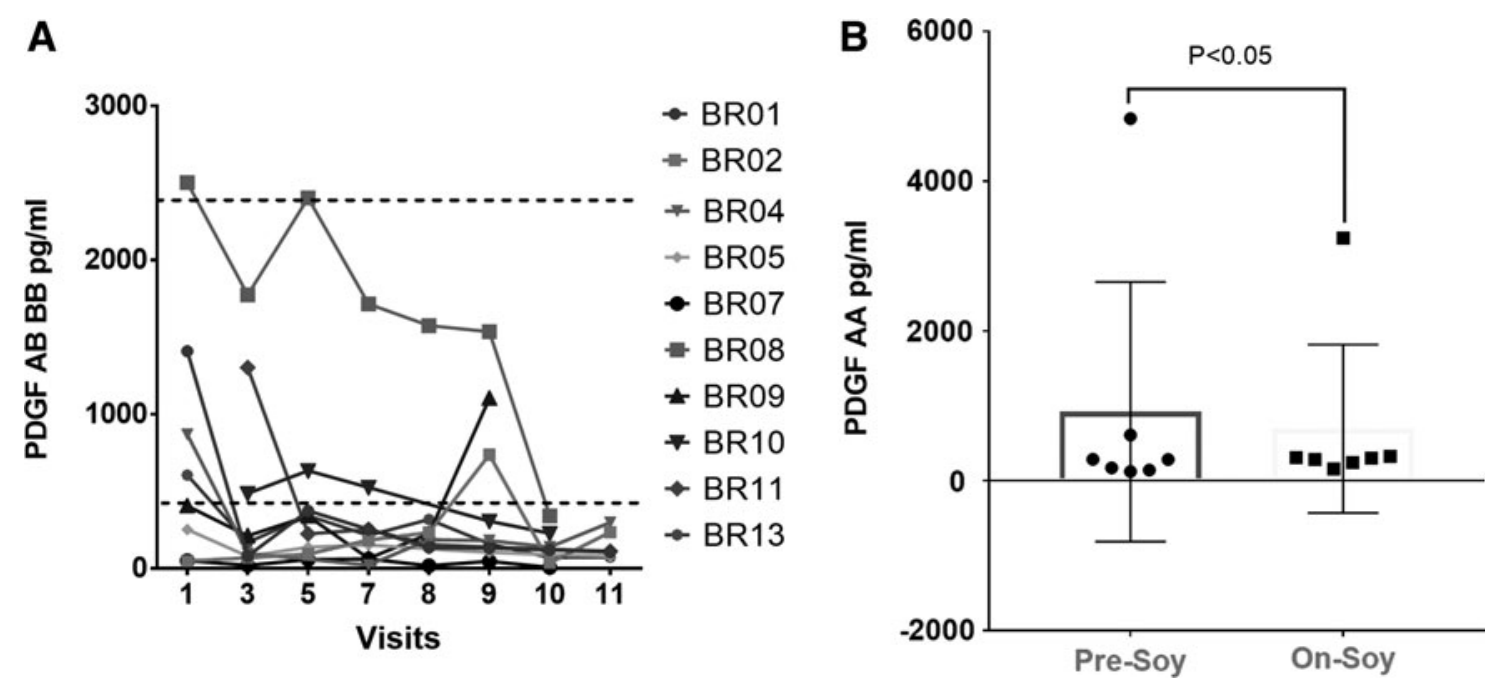

FIG. 3. (A) Plot graphs showing serum PDGF AA levels in obese subjects, pretreatment, and during treatment of Q-CAN Plus. (B) Histograms depicting mean \pm SD values of serum PDGF AA levels in obese subjects, pretreatment, and during treatment of Q-CAN Plus.

suggesting that it is a valid result. The value of this new data is it demonstrates the same beneficial effect from an easily available fermented soy product. In addition, an independent third-party chemical and microbial analysis of Q-CAN Plus found that the chemical composition of this commercial preparation was compliant with the manufacturer's certificate of analysis and is safe for human consumption. In conclusion, our preliminary study on Q-CAN Plus highlights its hypolipidemic effects and suppression of PDGF-AA in lean and obese subjects. Future studies need to confirm this effect, particularly in subjects with dyslipidemia, and to determine the mechanism of action.

\section{AUTHOR DISCLOSURE STATEMENT}

No competing financial interests exist.

\section{FUNDING INFORMATION}

This study was funded by a sponsored research agreement by BESO Biological Research, Inc. The company did not design the study, review the data, or contribute to the conclusions reached. Dr. Suyavaran Arumugam had full academic freedom to publish the data whether it was positive or negative.

\section{REFERENCES}

1. Assefa Y, Bajjalieh N, Archontoulis S, et al.: Spatial characterization of soybean yield and quality (amino acids, oil, and protein) for United States. Sci Rep 2018;8:14653.

2. Garcia MC, Torre M, Marina ML: Characterization of commercial soybean products by conventional and perfusion reversedphase high-performance liquid chromatography and multivariate analysis. J Chromatogr A 2000;881:47-57.
3. Kim JS, Lee JH, Surh J, Kang SA, Jang KH: Aglycone isoflavones and exopolysaccharides produced by Lactobacillus acidophilus in fermented soybean paste. Prev Nutr Food Sci 2016;21:117-123.

4. Liem IT, Steinkraus KH, Cronk TC: Production of vitamin B-12 in tempeh, a fermented soybean food. Appl Environ Microbiol 1977;34:773-776.

5. Denter J, Bisping B: Formation of B-vitamins by bacteria during the soaking process of soybeans for tempe fermentation. Int $J$ Food Microbiol 1994;22:23-31.

6. Ramdath DD, Padhi EM, Sarfaraz S, Renwick S, Duncan AM: Beyond the cholesterol-lowering effect of soy protein: A review of the effects of dietary soy and its constituents on risk factors for cardiovascular disease. Nutrients 2017;9:324.

7. Dong S, Zhang R, Ji YC, Hao JY, Ma WW, Chen XD, Xiao R, Yu HL: Soy milk powder supplemented with phytosterol esters reduced serum cholesterol level in hypercholesterolemia independently of lipoprotein E genotype: A random clinical placebocontrolled trial. Nutr Res 2016;36:879-884.

8. Lazarevic B, Boezelijn G, Diep LM, et al.: Efficacy and safety of short-term genistein intervention in patients with localized prostate cancer prior to radical prostatectomy: A randomized, placebo-controlled, double-blind Phase 2 clinical trial. Nutr Cancer 2011;63:889-898.

9. Hales CM, Fryar CD, Carroll MD, Freedman DS, Ogden CL: Trends in obesity and severe obesity prevalence in US youth and adults by sex and age, 2007-2008 to 2015-2016. JAMA 2018; 319:1723-1725.

10. Taku K, Umegaki K, Sato Y, Taki Y, Endoh K, Watanabe S: Soy isoflavones lower serum total and LDL cholesterol in humans: A meta-analysis of 11 randomized controlled trials. Am J Clin Nutr 2007;85:1148-1156.

11. Abderrahmani A, Yengo L, Caiazzo R, et al:: Increased hepatic PDGF-AA signaling mediates liver insulin resistance in obesityassociated type 2 diabetes. Diabetes 2018;67:1310-1321. 